\title{
Study on synchrony of two uncoupled neurons under the neuron's membrane potential stimulation
}

\author{
Yue-Ping Peng ${ }^{1,2}$, Jue Wang ${ }^{1}$, Quan-Xing Miao², Hong-Yan Lu ${ }^{2}$ \\ ${ }^{1}$ Key Laboratory of Biomedical Information Engineering of Education Ministry, Xi'an Jiaotong University, Xi'an, China; \\ ${ }^{2}$ Communication Department, Engineering College of Armed Police Force, Xi'an, China. \\ Email: percy001@163.com
}

Received 27 June 2009; revised 5 December 2009; accepted 7 December 2009.

\begin{abstract}
The input current of two uncoupled Hindmarsh-Rose neurons under different initial conditions is modulated by the membrane potential of the HindmarshRose neuron; and the synchronization characteristics of the two uncoupled neurons are discussed by analyzing their membrane potentials and their inter spike interval (ISI) distribution. Under the stimulation of the neuron's membrane potential whose discharge pattern is period or the chaos, the two uncoupled neurons under different initial conditions, whose parameter $r$ (the parameter $r$ is related to the membrane penetration of calcium ion, and reflects the changing speed of the slow adaptation current) is different or the same, can realize the full synchronization (state synchronization) or discharge synchronization (phase synchronization), and can only be synchronized to the discharge pattern of the stimulation neuron. The synchronization characteristics are mainly related to the discharge pattern and the strength of the stimulation neuron's membrane potential, and are little related to the parameter $r$ and the initial state of the two uncoupled neurons. This investigation shows the characteristics of the neuron's membrane potential affecting the synchronization process of neurons, and the neurons' discharge patterns and synchronization process can be adjusted and controlled by the discharge pattern and the strength of the stimulation neuron's membrane potential. This result is helpful to study synchronization and encode of many neurons or neural network.
\end{abstract}

Keywords: The Membrane Potential; The HR Neuron; Synchronization; ISI

\section{INTRODUCTION}

Since system synchronization was presented by Pecora,

This work is supported by the National Natural Science Foundation of China (No. 30670660). et al. [1,2] in 1991, synchronization research has been causing researchers' wide focus in neuroscience field. Nervous activities' synchronization is found not only among coupled neuron groups in the same brain region, but also among uncoupled neuron groups in the same brain region or among different cortical areas; Moreover, it can cross over two semispheres of the brain [3]. So in the nervous system, synchronization activities are presented not only among the coupled neurons, but also among the uncoupled neurons. Studies on neuron synchronization are mainly focused on two cases: the coupled neurons and the uncoupled neurons; and the synchronization of coupled neurons are studied more.

The Hindmarsh-Rose neuron (HR neuron) has several characteristics of the excitable cell's physical model and many time scale discharge action $[4,5,6,13]$. There are some literatures of studies on synchronization of $\mathrm{HR}$ neurons [3,7,9,10,12,14,15,16,17]. Huerta, R. and Rabinovich, M.I. studied the period rhythm's change of two HR neurons coupled by the circuit and the synapse [7]. Two uncoupled HR neurons can be realized synchronization by applying input signals to modulate the neuron model's parameter or by applying the noise and the HR neuron's membrane potential to stimulate these neurons $[8,9,10]$. The phase synchronization of two coupled HR neurons was discussed by Shuai, JianWei and Durand, D.M., and they concluded that the phase synchronization is the discharge synchronization, the frequency synchronization is the cluster synchronization, and the full synchronization is the state synchronization [11]. Synchrony of two uncoupled neurons under half wave sine current stimulation was discussed, and it was concluded that the two uncoupled HR neurons under different initial conditions, whose parameter $r$ is different or the same, can realize discharge synchronization (phase synchronization) or the full synchronization (state synchronization) [12].

In this study, we take two uncoupled HR neurons as the object, and make the HR neurons different initial discharge patterns by setting the value of the parameter $r$, and apply the membrane potential of the Hindmarsh- 
Rose neuron to modulate the two uncoupled HR neurons' input current, and discuss the HR neurons' synchronization characteristics by calculating and analyzing the two neurons' membrane potentials and their inter spike intervals (ISIs).

\section{THE DISCHARGE PATTERNS OF THE HR NEURON MODEL}

The HR neuron has many time scale dynamics action, and its equation is set of three dimension ordinary differential equations $[4,5,6]$ :

$$
\begin{aligned}
& x=y-a x^{3}+b x^{2}-z+I \\
& y=c-d x^{2}-y \\
& z=r[s(x-X)-z]
\end{aligned}
$$

The HR neuron has three time variables: the membrane potential variable $x$ which has the quick depolarization ability, the quick recovery variable $y$ and slow adaptation current variable $z . I$ is the input stimulation current; $a, b, c, d, s, r$ and $X$ are parameters. The parameter $r$ is related to the membrane penetration of calcium ion, and reflects the changing speed of the slow adaptation current variable $z$. Other parameters have no specific physical meaning. The equations are nondimensional, and at numerical calculation, value of parameters is as follows: $a=1.0, b=3.0, c=1.0, d=5.0, s=4.0, I=3.0$, $X=-1.56$, and you can set the neuron at different discharge patterns by changing the parameter $r$.

The discharge threshold value is -0.25 , and if the membrane potential is more than -0.25 , the neuron will produce one discharge process. Figure 1 is the ISI bifurcation figure of the neuron, where parameters except $r$ are set to the above values, and the initial state of the neuron is $(1.0,0.2,0.2)$. From Figure 1, the discharge pattern of the neuron begins from the chaos $\operatorname{state}(r$ is

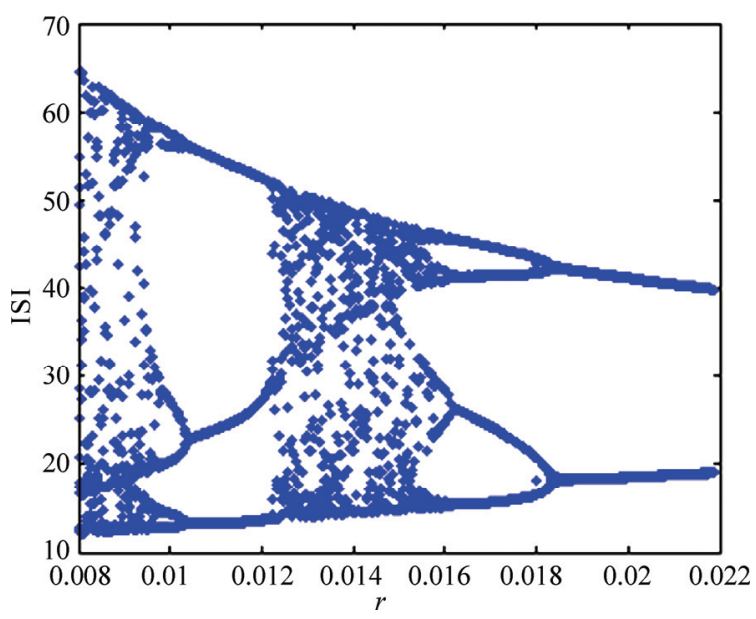

Figure 1. Bifurcation figure of the HR neuron under the parameter $r$ changing from 0.008 to 0.022 .
Table 1. The values of the two neurons' parameter $r$.

\begin{tabular}{cc}
\hline Case & The values of $r_{1}$ and $r_{2}$ \\
\hline \multirow{3}{*}{ The same } & $r_{1}=r_{2}=0.009, r_{1}=r_{2}=0.011$, \\
& $r_{1}=r_{2}=0.013, r_{1}=r_{2}=0.0171, r_{1}=r_{2}=0.02$ \\
& $r_{1}=0.0085$ and $r_{2}=0.009$, \\
& $r_{1}=0.011$ and $r_{2}=0.0115$, \\
& $r_{1}=0.014$ and $r_{2}=0.0141$, \\
& $r_{1}=0.017$ and $r_{2}=0.0171$, \\
& $r_{1}=0.021$ and $r_{2}=0.0215$ \\
& $r_{1}=0.0082$ and $r_{2}=0.0115$, \\
& $r_{1}=0.014$ and $r_{2}=0.0085$, \\
& $r_{1}=0.0085$ and $r_{2}=0.017$, \\
& $r_{1}=0.009$ and $r_{2}=0.021$, \\
& $r_{1}=0.011$ and $r_{2}=0.0145$, \\
& $r_{1}=0.0112$ and $r_{2}=0.017$, \\
& $r_{1}=0.0115$ and $r_{2}=0.021$, \\
& $r_{1}=0.014$ and $r_{2}=0.017$, \\
& $r_{1}=0.022$ and $r_{2}=0.013$, \\
& $r_{1}=0.017$ and $r_{2}=0.021$ \\
\hline
\end{tabular}

about 0.008 0.009), and evolves period 6 discharge pat$\operatorname{tern}(r$ is near 0.01$)$, and via the adverse period doubling bifurcation passes period $3(r$ is about $0.0105 \sim 0.012)$ and enters the chaos $\operatorname{state}(r$ is about $0.0125 \sim 0.015)$ again, and at last via the adverse period doubling bifurcation passes period $4(r$ is about $0.016 \sim 0.018)$ and comes into period 2 ( $r$ is about $0.0185 \sim 0.022$ ).

\section{THE SYNCHRONIZATION OF TWO UNCOUPLED HR NEURONS UNDER THE STIMULATION OF THE NEURON'S MEMBRANE POTENTIAL}

The equation set of two uncoupled HR neurons' model is:

$$
\begin{aligned}
& \dot{x}_{i}=y_{i}-a_{i} x_{i}^{3}+b_{i} x_{i}^{2}-z_{i}+\left(I_{i}+I_{S}(t)\right) \\
& \dot{y}_{i}=c_{i}-d_{i} x_{i}^{2}-y_{i} \\
& \dot{z}_{i}=r_{i}\left[s_{i}\left(x_{i}-X_{i}\right)-z_{i}\right] \quad(i=1,2)
\end{aligned}
$$

Time variables of these two neurons are respectively $\left(x_{1}, y_{1}, z_{1}\right)$ and $\left(x_{2}, y_{2}, z_{2}\right)$, and the parameters of these two neurons are respectively $\left(a_{1}, b_{1}, c_{1}, d_{1}, r_{1}, s_{1}, I_{1}, X_{1}\right)$ and $\left(a_{2}, b_{2}, c_{2}, d_{2}, r_{2}, s_{2}, I_{2}, X_{2}\right)$.

The equations are also nondimensional. At numerical calculation, the values of these two neurons' parameters (except $r$ ) are as follows: $a_{1}=a_{2}=1.0, b_{1}=b_{2}=3.0$, $c_{1}=c_{2}=1.0, d_{1}=d_{2}=5.0, s_{1}=s_{2}=4.0, X_{1}=X_{2}=-1.56, I_{1}=I_{2}=3.0$; and the values of the initial states of these two neurons are respectively $(1.0,0.2,0.2)$ and $(-1.0,0.8,0.3)$; and you can set these two neurons at different discharge patterns by controlling values of the parameter $r_{\mathrm{i}}(\mathrm{i}=1,2)$.

According to the difference of these two neurons' parameter $r$, there are three cases: the parameter $r$ is the same, a little different $\left(\left|r_{1}-r_{2}\right| \leq 0.0005\right)$, and much 
different $\left(\left|r_{1}-r_{2}\right| \geq 0.003\right)$. Table 1 shows the values of the two neurons' parameter $r$, and the discharge patterns of these two neurons are correspondingly showed in the Figure 1.

The value of the initial state of the stimulation neuron is $(0.2,1,-0.2)$. The values of the parameters except $r$ of the stimulation neuron are as follows: $a=1.0, b=3.0$, $c=1.0, d=5.0, s=4.0, I=3.0, X=-1.56$. The stimulation neuron can be made different discharge patterns by changing the parameter $r$ according to Figure 1 .

Total stimulation current includes two parts: the bias current $\left(I_{1}\right.$ and $\left.I_{2}\right)$ and the input stimulation current $I_{\mathrm{S}}(\mathrm{t})$ : $I_{S}(t)=k x(t) \cdot x(\mathrm{t})$ is the membrane potential of the stimulation neuron, and $k$ is the stimulation strength of the membrane potential. The stimulation neuron begins to stimulate the system model after the bias current $\left(I_{1}\right.$ and $I_{2}$ ) has been working for 500 . The simulation time of the system model is often $0 \sim 4000$.

\subsection{Synchronization under the Stimulation of the Period Discharge Pattern}

The values of the parameters of these two uncoupled neurons are above given values. The parameter $r$ of the stimulation neuron is respectively $0.011,0.017$, and 0.02 , and its discharge pattern is respectively period 3 , period 4, and period 2. Under each discharge pattern, the stimulation strength $k$ is respectively $0.5,1,2,3,6,9$, and 12 .

The parameter $r$ of the stimulation neuron is 0.011 (the discharge pattern is period 3). As it begins to stimulate the two neurons, in above all cases, the two uncouple neurons' discharge patterns totally change into dynamic period discharge pattern from the initial discharge patterns with the stimulation strength $k$ increasing, and the changing trend of their membrane potentials' ISI is the same, namely gradually evolves into period 3 discharge pattern which is the same as the stimulation neuron's. When the stimulation strength is 0.5 and 1 , these two neurons don't realize synchronization, and when the stimulation strength is $3,6,9$ and 12 , these two neurons realize discharge synchronization (phase synchronization) or the full synchronization (state synchronization) [11]. When the stimulation strength is 2 , only several groups of these two neurons realize discharge synchronization or the full synchronization. When the parameter $r$ is the same, these two neurons realize the full synchronization. When the parameter $r$ is a little different, these two neurons realize the full synchronization or discharge synchronization. When the parameter $r$ is much different, these two neurons realize discharge synchronization or the approximate full synchronization. The synchronization discharge pattern is the same as the stimulation neuron's discharge pattern (period 3).

The parameter $r$ of the stimulation neuron is 0.017 (the discharge pattern is period 4). As it begins to stimu- late the two neurons, in above all cases, the two uncouple neurons' discharge patterns totally change into dynamic period discharge pattern from the initial discharge patterns with the stimulation strength $k$ increasing, and the changing trend of their membrane potentials' ISI is the same, namely gradually evolves into period 4 discharge pattern which is the same as the stimulation neuron's. When the stimulation strength is 0.5 and 1 , these two neurons don't realize synchronization, and when the stimulation strength is $3,6,9$ and 12 , these two neurons realize discharge synchronization or the full synchronization. When the stimulation strength is 2 , only several groups of these two neurons realize discharge synchronization or the full synchronization. When the parameter $r$ is the same, these two neurons realize the full synchronization. When the parameter $r$ is a little different, these two neurons realize the full synchronization or discharge synchronization. When the parameter $r$ is much different, these two neurons realize discharge synchronization or the approximate full synchronization. The synchronization discharge pattern is the same as the stimulation neuron's discharge pattern (period 4).

The parameter $r$ of the stimulation neuron is 0.02 (the discharge pattern is period 2). As it begins to stimulate the two neurons, in above all cases, the two uncouple neurons' discharge patterns totally change into dynamic period discharge pattern from the initial discharge patterns with the stimulation strength $k$ increasing, and the changing trend of their membrane potentials' ISI is the same, namely gradually evolves into period 2 discharge pattern which is the same as the stimulation neuron's. When the stimulation strength is 0.5 and 1 , these two neurons don't realize synchronization, and when the stimulation strength is 6,9 and 12 , these two neurons realize discharge synchronization or the full synchronization. When the stimulation strength is 2 or 3 , only several groups of these two neurons realize discharge synchronization or the full synchronization. When the parameter $r$ is the same, these two neurons realize the full synchronization. When the parameter $r$ is a little different, these two neurons realize the full synchronization or discharge synchronization. When the parameter $r$ is much different, these two neurons realize discharge synchronization or the approximate full synchronization. The synchronization discharge pattern is the same as the stimulation neuron's discharge pattern (period 2).

Limited to the length, Figure 2 only shows the synchronization process of the two neurons, where the parameter $r$ of the stimulation neuron is 0.02 , and the stimulation strength $k$ is 9. In Figure 2, from (a) and (d), (b) and (e), (c) and (f), these two neurons respectively realize the full synchronization, the full synchronization, and the discharge synchronization; and the synchronization discharge pattern is all period 2, which is the same as the stimulation neuron's discharge pattern. 

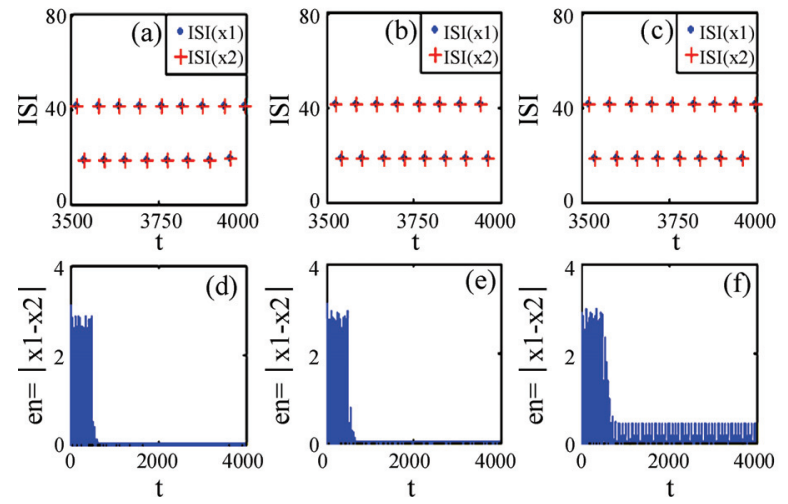

Figure 2. The synchronization process of the two neurons, where the parameter $r$ of the stimulation neuron is 0.02 and the stimulation strength is 9. (a) The membrane potential's ISI changing chart of the two neurons with time, where $r_{l}$ is 0.013 and $r_{2}$ is 0.013 . (b) The membrane potential's ISI changing chart of the two neurons with time, where $r_{l}$ is 0.014 and $r_{2}$ is 0.0141 . (c) The membrane potential's ISI changing chart of the two neurons with time, where $r_{l}$ is 0.022 and $r_{2}$ is 0.013 . (d) The two neurons' membrane potential's synchronization error (err $\left.=\left|x_{1}-x_{2}\right|\right)$ changing chart with time, where $r_{1}$ is 0.013 and $r_{2}$ is 0.013 . (e) The two neurons' membrane potential's synchronization error changing chart with time, where $r_{1}$ is 0.014 and $r_{2}$ is 0.0141 . (f) The two neurons' membrane potential's synchronization error changing chart with time, where $r_{l}$ is 0.022 and $r_{2}$ is 0.013 .

Synchronization state of two neurons can also be classified by the phase function [11]. The phase function of these two neurons is here defined as: $\varphi_{i}(t)=$ $\arctan \left[\dot{x}_{i}(t-0.5) /\left(\dot{x}_{i}(t)+0.1\right)\right] \quad(\mathrm{i}=1,2)$, where $\mathrm{x}(\mathrm{t})$ is the membrane potential. Limited to the length, Figure 3 only shows the trajectory projection in the $\dot{x}_{i}(t-0.5)-\left(\dot{x}_{i}(t)+0.1\right)$ plane and the phase of the neuron changing with time. From Figures 3(a), 3(b) and $3(\mathrm{c})$, it can be seen that the attractor has a single rotation center in the $\dot{x}_{i}(t-0.5)-\left(\dot{x}_{i}(t)+0.1\right)$ plane. The phases of the neuron are given in Figure 3(g), 3(h) and 3(i) with time from 3500 to 4000 . The corresponding membrane potentials of the neuron are given in Figure 3(d), 3(e) and 3(f).

The absolute phase difference of two neurons $(|\Delta \phi(t)|)$ is defined as: $|\Delta \phi(t)|=\left|\phi_{1}(t)-\phi_{2}(t)\right|$. When the maximum absolute phase difference of these two neurons $\left(|\Delta \phi(t)|_{\max }\right)$ is no more than $2 \pi$, these two neurons realize discharge synchronization; When $|\Delta \phi(t)|_{\max }$ approximates to zero, these two neurons realize the full synchronization; when $|\Delta \phi(t)|_{\max }$ can be as large as $4 \pi$ for some small time intervals, these two neurons can be viewed as an intermittent discharge synchronization.

Limited to the length, Figure 4 only partly shows the
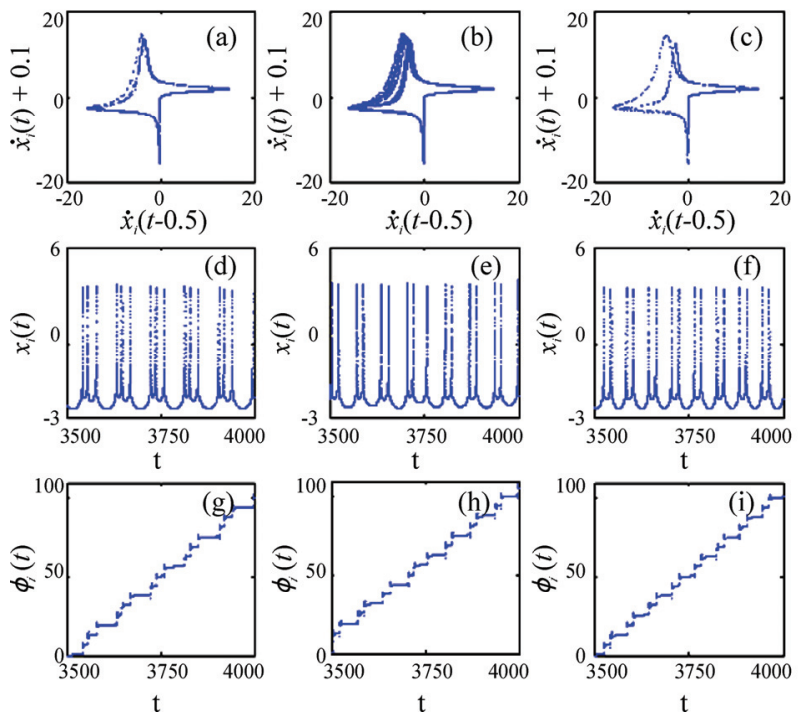

Figure 3. The trajectory projection in the $\dot{x}_{i}(t-0.5)-\left(\dot{x}_{i}(t)+0.1\right)$ plane and the phase of neuron changing with time, where the strength $k$ of the stimulation neuron is 9. (a) The trajectory projection in the plane of the neuron, whose parameter $r$ is 0.021 , where the parameter $r$ of the stimulation neuron is 0.011 . (b) The trajectory projection in the plane of the neuron, whose parameter $r$ is 0.009 , where the parameter $r$ of the stimulation neuron is 0.013 . (c) The trajectory projection in the plane of the neuron, whose parameter $r$ is 0.011 , where the parameter $r$ of the stimulation neuron is 0.02 . (d) The membrane potential changing chart with time of the neuron, whose parameter $r$ is 0.021 , where the parameter $r$ of the stimulation neuron is 0.011 . (e) The membrane potential changing chart with time of the neuron, whose parameter $r$ is 0.009 , where the parameter $r$ of the stimulation neuron is 0.013 . (f) The membrane potential changing chart with time of the neuron, whose parameter $r$ is 0.011 , where the parameter $r$ of the stimulation neuron is 0.02 . (g) The phase function changing chart with time of the neuron, whose parameter $r$ is 0.021 , where the parameter $r$ of the stimulation neuron is 0.011 . (h) The phase function changing chart with time of the neuron, whose parameter $r$ is 0.009 , where the parameter $r$ of the stimulation neuron is 0.013 . (i) The phase function changing chart with time of the neuron, whose parameter $r$ is 0.011 , where the parameter $r$ of the stimulation neuron is 0.02 .

changing diagram of $|\Delta \phi(t)|_{\max }$ with the stimulation strength $k$ changing, where the parameter $r$ of the stimulation neuron is 0.02 . From Figure 4, when the stimulation strength $k$ changes from 0 to 8 according to the step 0.02 , these two neurons' discharge patterns begin with asynchronization, and gradually realize discharge synchronization or the full synchronization via the quick intermittent discharge synchronization process. When the stimulation strength $k$ is near 2.6, and these two neurons in Figure 4(a) go into the intermittent discharge synchronization state, so the critical value of these two neurons realizing synchronization is about 2.6. When the stimulation strength $k$ is near 2.1, and these two neurons 
in Figure 4(b) go into the intermittent discharge synchronization state, so the critical value of these two neurons realizing synchronization is about 2.1. When the stimulation strength $k$ is near 2.5 , and these two neurons in Figure 4(c) go into the intermittent discharge synchronization state, so the critical value of these two neurons realizing synchronization is about 2.5. When the stimulation strength $k$ is near 2, and these two neurons in Figure 4(d) go into the intermittent discharge synchronization state, so the critical value of these two neurons realizing synchronization is about 2 . When the stimulation strength $k$ is near 2.9, and these two neurons in Figure 4(e) go into the intermittent discharge synchronization state, so the critical value of these two neurons realizing synchronization is about 2.9. So, under the same stimulation signal, the stimulation strength's critical value of the two neurons whose parameter $r$ is at $0.008 \sim 0.009$, is bigger than that of the two neurons whose parameter $r$ is at $0.0125 \sim 0.015$. Under the same stimulation signal, the less the difference between the two neurons is the smaller stimulation strength $k$ of the two neurons' realizing synchronization and the easier the two neurons realize synchronization. In addition, the bigger the stimulation strength $k$ is easier the neurons realize synchronization.

\subsection{Synchronization under the Stimulation of the Chaos Discharge Pattern}

The values of the parameters of these two uncoupled neurons are above given values. The parameter $r$ of the stimulation neuron is respectively 0.0085 and 0.013 , and its discharge pattern is the chaos. Under each parameter $r$, the stimulation strength $k$ is respectively $0.5,1,2,3,6$, 9 , and 12 .

The parameter $r$ of the stimulation neuron is 0.0085 (the discharge pattern is the chaos). As it begins to stimulate the two neurons, in above all cases, the two uncouple neurons' discharge patterns totally change into dynamic period discharge pattern from the initial discharge patterns with the stimulation strength $k$ increasing, and the changing trend of their membrane potentials' ISI is the same, namely gradually evolves into the chaos discharge pattern which is the same as the stimulation neuron's. When the stimulation strength is 0.5 and 1 , these two neurons don't realize synchronization, and when the stimulation strength is 3,6,9 and 12, these two neurons realize discharge synchronization or the full synchronization. When the stimulation strength is 2 , these two neurons except $r_{1}=0.009$ and $r_{2}=0.021, r_{1}=$ 0.022 and $r_{2}=0.013$, realize discharge synchronization or the full synchronization. When the parameter $r$ is the same, these two neurons realize the full synchronization. When the parameter $r$ is a little different, these two neurons realize the full synchronization or discharge synchronization. When the parameter $r$ is much different, these two neurons realize discharge synchronization or the approximate full synchronization. The synchronization discharge pattern is the same as the stimulation neuron's discharge pattern (the chaos).

The parameter $r$ of the stimulation neuron is 0.013 (the discharge pattern is the chaos). As it begins to stimulate the two neurons, in above all cases, the two uncouple neurons' discharge patterns totally change into dynamic period discharge pattern from the initial discharge patterns with the stimulation strength $k$ increasing, and the changing trend of their membrane potentials' ISI is the same, namely gradually evolves into the chaos discharge pattern which is the same as the stimulation neuron's. When the stimulation strength is 0.5 and 1 , these two neurons don't realize synchronization, and when the stimulation strength is $3,6,9$ and 12 , these two neurons realize discharge synchronization or the full synchronization. When the stimulation strength is 2 , only several groups of these two neurons realize discharge synchronization or the full synchronization. When the parameter $r$ is the same, these two neurons realize the full synchronization. When the parameter $r$ is a little different, these two neurons realize the full synchronization or discharge synchronization. When the parameter $r$ is much different, these two neurons realize discharge synchronization or the approximate full synchronization. The synchronization discharge pattern is
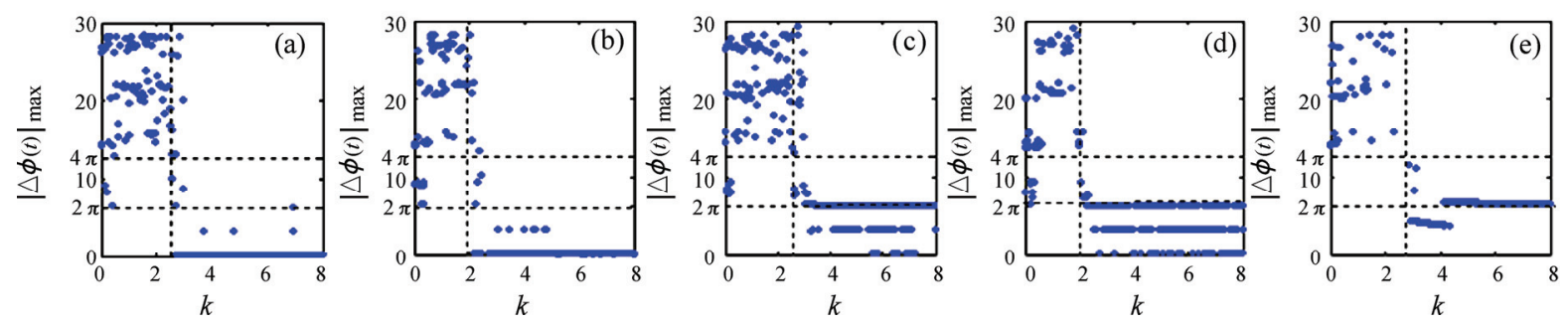

Figure 4. The changing diagram of $|\Delta \phi(t)|_{\max }$ is changing with the stimulation strength $k$ changing, where the parameter $r$ of

the stimulation neuron is 0.02 , and the changing step of the stimulation strength $k$ is 0.02 . (a) The parameter $r$ of these two neurons is the same: $r_{l}=r_{2}=0.009$. (b) The parameter $r$ of these two neurons is the same: $r_{I}=r_{2}=0.013$. (c) The parameter $r$ of these two neurons is a little different: $r_{l}=0.0085$ and $r_{2}=0.009$. (d) The parameter $r$ of these two neurons is a little different: $r_{1}=0.014$ and $r_{2}=0.0141$. (e) The parameter $r$ of these two neurons is much different: $r_{l}=0.014$ and $r_{2}=0.0085$. 

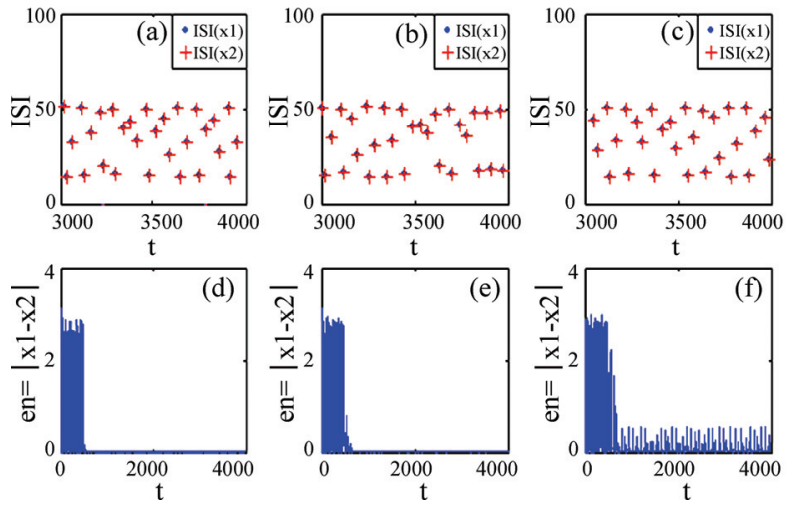

Figure 5. The synchronization process of the two neurons, where the parameter $r$ of the stimulation neuron is 0.013 and the stimulation strength $k$ is 9. (a) The membrane potential's ISI changing chart of the two neurons with time, where $r_{l}$ is 0.013 and $r_{2}$ is 0.013 . (b) The membrane potential's ISI changing chart of the two neurons with time, where $r_{l}$ is 0.014 and $r_{2}$ is 0.0141 . (c) The membrane potential's ISI changing chart of the two neurons with time, where $r_{l}$ is 0.022 and $r_{2}$ is 0.013 . (d) The two neurons' membrane potential's synchronization error $\left(\right.$ err $\left.=\left|x_{1}-x_{2}\right|\right)$ changing chart with time, where $r_{1}$ is 0.013 and $r_{2}$ is 0.013 . (e) The two neurons' membrane potential's synchronization error changing chart with time, where $r_{l}$ is 0.014 and $r_{2}$ is 0.0141 . (f) The two neurons' membrane potential's synchronization error changing chart with time, where $r_{1}$ is 0.022 and $r_{2}$ is 0.013 .

the same as the stimulation neuron's discharge pattern (the chaos).

Limited to the length, Figure 5 only shows the synchronization process of the two neurons. In Figure 5, from (a) and (d), (b) and (e), (c) and (f), these two neurons respectively realize the full synchronization, the full synchronization, and the discharge synchronization; and the synchronization discharge pattern is all the chaos, which is the same as the stimulation neuron's discharge pattern.

Limited to the length, Figure 6 only shows the changing diagram of $|\Delta \phi(t)|_{\max }$ with the stimulation strength $k$ changing. From Figure 6, when the stimulation strength $k$ changes from 0 to 8 according to the step 0.02 , these two neurons' discharge patterns begin with asynchronization, and gradually realize discharge synchronization or the full synchronization via the quick intermittent discharge synchronization process.

When the stimulation strength $k$ is near 1.7, and these two neurons in Figure 6(a) go into the intermittent discharge synchronization state, so the critical value of these two neurons realizing synchronization is about 1.7. When the stimulation strength $k$ is near 1.3 , and these two neurons in Figure 6(b) go into the intermittent discharge synchronization state, so the critical value of these two neurons realizing synchronization is about 1.3. When the stimulation strength $k$ is near 1.6 , and these two neurons in Figure 6(c) go into the intermittent discharge synchronization state, so the critical value of these two neurons realizing synchronization is about 1.6. When the stimulation strength $k$ is near 1.4 , and these two neurons in Figure 6(d) go into the intermittent discharge synchronization state, so the critical value of these two neurons realizing synchronization is about 1.4. When the stimulation strength $k$ is near 1.8 , and these two neurons in Figure 6(e) go into the intermittent discharge synchronization state, so the critical value of these two neurons realizing synchronization is about 1.8 . So, under the same stimulation signal, the stimulation strength's critical value of the two neurons whose parameter $r$ is at $0.008 \sim 0.009$, is bigger than that of the two neurons whose parameter $r$ is at $0.0125 \sim 0.015$. Under the same stimulation signal, the less the difference between the two neurons is, the smaller the stimulation strength $k$ of the two neurons' realizing synchronization is, and the easier the two neurons realize synchronization. From Figures 4 and 6, for the two neurons, the critical value of the chaos signal's stimulation strength is less than that of the period signal's stimulation strength, so the chaos signal's stimulation is easier to realize the synchronization of two neurons than the period signal's stimulation. In addition, the bigger the stimulation strength $k$ is, the easier the neurons realize synchronization.
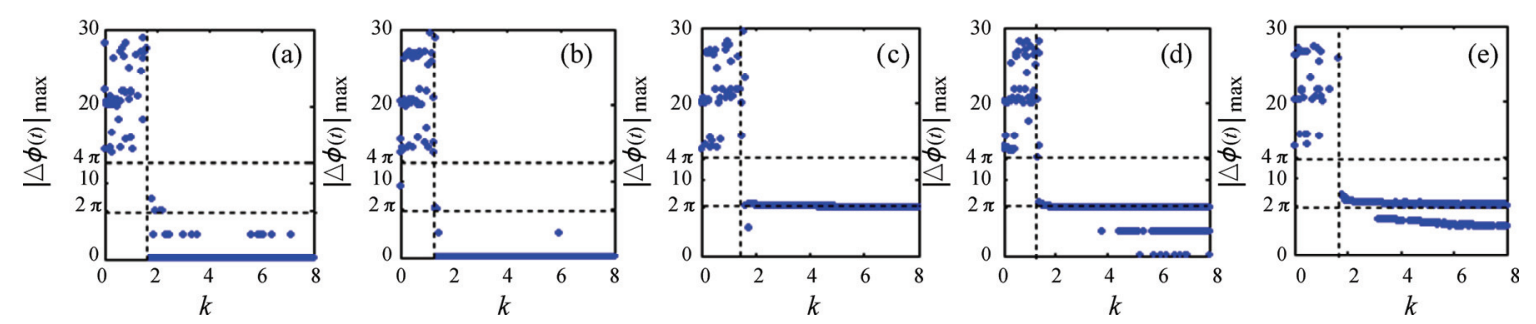

Figure 6. The changing diagram of $|\Delta \phi(t)|_{\max }$ is changing with the stimulation strength $k$, where the parameter $r$ of the stimulation neuron is 0.0085 , and the changing step of the stimulation strength $k$ is 0.02 . (a) The parameter $r$ of these two neurons is the same: $r_{1}=r_{2}=0.009$. (b) The parameter $r$ of these two neurons is the same: $r_{1}=r_{2}=0.013$. (c) The parameter $r$ of these two neurons is a little different: $r_{1}=0.0085$ and $r_{2}=0.009$. (d) The parameter $r$ of these two neurons is a little different: $r_{l}=0.014$ and $r_{2}=0.0141$. (e) The parameter $r$ of these two neurons is much different: $r_{I}=0.014$ and $r_{2}=0.0085$. 


\section{CONCLUSIONS}

Under the stimulation of the neuron's membrane potential whose discharge pattern is period or the chaos, the two uncoupled neurons under different initial conditions, whose parameter $r$ is different or the same, can realize the full synchronization or discharge synchronization, and can only be synchronized to the response being in synchronization with stimulation signal. The synchronization discharge patterns are the same as the stimulation neuron, and the synchronization characteristics are mainly related to the discharge pattern and the strength of the stimulation neuron's membrane potential, and are little related to the parameter $r$ and the initial state of the two uncoupled neurons. And the bigger the strength of the stimulation signal is, the easier the two neurons realize synchronization. The critical strength value of these two neurons realizing synchronization is related to, not only the two neurons' parameter $r$ and the initial state, but also the stimulation signal. For the two neurons, the critical value of the chaos signal's stimulation strength is less than that of the period signal's stimulation strength. The chaos signal's stimulation is easier to realize synchronization of two neurons than the period signal's stimulation.

This investigation shows characteristics of the neuron's membrane potential affecting the synchronization process of two uncoupled neurons, and the two neurons' discharge patterns and synchronization process can be adjusted and controlled by the discharge pattern and the strength of the stimulation neuron's membrane potential. This result is helpful to study synchronization and encode of many neurons or neural network.

\section{ACKNOWLEDGEMENTS}

The authors would like to thank "The Journal of Biomedical Science and Engineering" for providing the chance and really appreciate the anonymous reviewers for the valuable comments on the paper.

\section{REFERENCES}

[1] Pecora, L.M. and Carroll, T.L. (1990) Synchronization in chaotic systems. Physical Review Letter, 64, 821-824.

[2] Pecora, L.M. and Carroll, T.L. (1991) Driving systems with chaotic signals. Physical Review: A, 44, 2374-2383.

[3] Gray, C.M. et al. (1989) Oscillatory response in cat visual cortex exhibit inter-columnar synchronization which reflects global stimulus properties. Nature, 338, 334-337.

[4] Hindmarsh, J.L. and Rose, R.M. (1982) A model of the nerve impulse using two first-order differential equation. Nature, 296, 162-165.

[5] Hindmarsh, J.L. and Rose, R.M. (1984) A Model of neuronal bursting using three coupled first order differential equations. Series B, Biological Sciences, 221, 87-102.

[6] Peng, Y.P., Jian, Z. and Wang, J. (2006) Study on discharge patterns of Hindmarsh-Rose neurons under slow wave current stimulation. Lecture Notes in Computer Science, 4221, 127-134.

[7] Huerta, R. and Rabinovich, M.I. (1997) Spike-train bifurcation in two coupled chaotic neurons. Physical Review: E, 55, R2108-R2110.

[8] Wu, Y. et al. (2005) Study on nonlinear characteristic of two synchronizing uncoupled Hindmarsh-Rose neurons. Physics Letters: A, 54, 3457-3464.

[9] He, D.H., Shi, P.L. and Stone, L. (2003) Noise-induced synchronization in realistic models. Physical Review: E, 67, 0272011-0272013.

[10] Wu, Y. et al. (2005) Complete synchronization and phase synchronization of two uncoupled neurons through parametrical drive. Journal of Xi' an Jiaotong University, 39, 544-547.

[11] Shuai, J.W. and Durand, D.M. (1999) Phase synchronization in two coupled chaotic neurons. Physics Letters: A, 264, 289-296.

[12] Peng, Y.P., Wang, J. and Jian, Z. (2009) Synchrony of two uncoupled neurons under half wave sine current stimulation. Communications in Nonlinear Science and Numerical Simulation, 14, 1570-1575.

[13] Peng, Y.P. and Wang, J. (2007) Discharge patterns' change of Hindmarsh-Rose neurons under slow ramp current stimulation. BIC-TA IEEE, 52-55.

[14] Shi, X. and Lu, Q.S. (2005) Coherence resonance and synchronization of Hindmarsh-Rose neurons with noise. Chinese Physics, 14(6), 1088-1094.

[15] Wu, Y., Xu, J.X., He, D.H. and David, J.D. Earn (2005) Generalized synchronization induced by noise and parameter mismatching in Hindmarsh-Rose neurons. Chaos, Solitons and Fractals, 23, 1605-1611.

[16] Katsutoshi, Y., Keijin, S. and Atsushi, S. (2006) Noiseinduced synchronization of uncoupled nonlinear systems. Journal of Sound and Vibration, 290, 34-47.

[17] Wu, Y., Xu, J.X., Jin, W.Y. and Hong, L. (2007) Detection of mechanism of noise-induced synchronization between two identical uncoupled neurons. Chin. Phys. Lett, 24(11), 3066-3069. 